\title{
Diffusion in spatially and temporarily inhomogeneous media
}

\author{
H. Lehr ${ }^{*}$ and F. Sagués \\ Departament de Química Física, Universitat de Barcelona, 08028 Barcelona, Spain \\ J. M. Sancho \\ Departament d'Estructura i Constituents de la Materia, Universitat de Barcelona, 08028 Barcelona, Spain
}

(Received 30 April 1996)

\begin{abstract}
In this paper we consider diffusion of a passive substance $C$ in a temporarily and spatially inhomogeneous two-dimensional medium. As a realization for the latter we choose a phase-separating medium consisting of two substances $A$ and $B$, whose dynamics is determined by the Cahn-Hilliard equation. Assuming different diffusion coefficients of $C$ in $A$ and $B$, we find that the variance of the distribution function of the said substance grows less than linearly in time. We derive a simple identity for the variance using a probabilistic ansatz and are then able to identify the interface between $A$ and $B$ as the main cause for this nonlinear dependence. We argue that, finally, for very large times the here temporarily dependent diffusion "constant" goes like $t^{-1 / 3}$ to a constant asymptotic value $D_{\infty}$. The latter is calculated approximately by employing the effective-medium approximation and by fitting the simulation data to the said time dependence. [S1063-651X(96)02111-3]
\end{abstract}

PACS number(s): 64.60.My, 47.27.Sd

\section{INTRODUCTION}

Needless to say, diffusion is a very important physical process and of major practical interest in many fields of science, ranging from statistical optics to diffusion controlled chemical reactions. Usually, when treating diffusion in liquids, one considers the medium to be homogeneous, although numerous examples exist where this assumption is not appropriate.

In this contribution we would like to study the case where the medium is no longer homogeneous, but has dynamically evolving inhomogeneities. We have opted here to use as a medium a solution of a dynamical equation corresponding to a binary alloy phase separation problem, i.e. in this case the well-known Cahn-Hilliard equation [1,2] (see, e.g., [3-5] for more recent literature on the theoretical aspects and [6-8] on the applied aspects of this subject). This equation describes the phase separation following a quench of a nonmiscible binary mixture (with phases $A$ and $B$ ) inside its coexistence curve. It is known (see, e.g., [9] and references therein) that the solutions to this equation are very structured, their configuration depending on the relative concentration of the phases.

Despite the importance of the actual physical situation that leads to the Cahn-Hilliard equation, it will serve here more as a model for a dynamically evolving inhomogeneous medium. Because of the properties mentioned it appears to be an ideal candidate because we are able to study the diffusion in a rather rigidly structured medium.

The idea for the study is now as follows. We assume that the scalar $C$ has different nonvanishing diffusion coefficients in the phases $A$ and $B$. If we mix these components the

\footnotetext{
*Permanent address: I.N. Stranski Insitut, Sekretariat ER 1, Technische Universität Berlin, Straße d. 17, Juni 112, D-10 623 Berlin, Germany.
}

diffusion coefficient at every space and time point will be proportional to the amount of phase $A$ and phase $B$ present at this point. Now, we let these concentrations (or rather the difference in molar fraction) evolve in time according to the Cahn-Hilliard equation. The question of interest then is the temporal development of the mean-square displacement of the dispersed scalar.

Therefore let us call $\chi$ the variable that according to the Cahn-Hilliard equation describes the temporal development of the difference in molar fraction

$$
\frac{\partial \chi}{\partial t}=\nabla^{2}\left(-\chi+\chi^{3}-\nabla^{2} \chi\right)
$$

with initial conditions being

$$
\chi(r, 0)=\chi_{0}+\alpha .
$$

$\alpha$ is a (uniform) random variable, whose actual range is not of critical importance as long as its average vanishes. Here we have chosen $\alpha \in[-0.1,0.1] \cdot \chi_{0}$ is the average difference in mole fraction. This average is an important parameter insofar as it determines the configuration of the appearing structures or inhomogeneities. So it is known, e.g., that for $\chi_{0}=0.4(-0.4)$ droplets of $B(A)$ in $A(B)$ appear, while for $\chi_{0}=0$ one finds lamellar structures; see Fig. 1. The equilibrium stable states of this differential equation lie at $\chi= \pm 1$.

Figure 1 shows these three cases. Figure 1(a) depicts the choice $\chi_{0}=-0.4$, Fig. 1(b) $\chi_{0}=0.0$, and Fig. 1(c) $\chi_{0}=0.4$ at time $t=200$. The average size of the appearing structures grows according to the Lifshitz-Slyozov time law [2], i.e., with $t^{1 / 3}$. Due to the conservative nature of Eq. (1), though, the total area occupied by the structures is constant. This means that the number of the structures growing with $t^{1 / 3}$ has to decrease with $t^{-2 / 3}$. Considering now the interface (i.e., the borders between zones of positive and negative $\chi$ ), one 


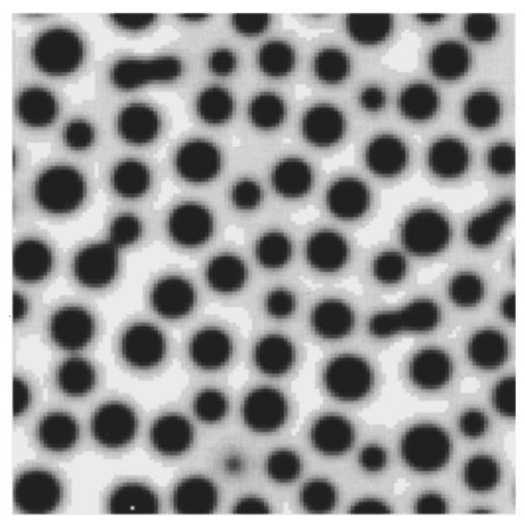

(a)

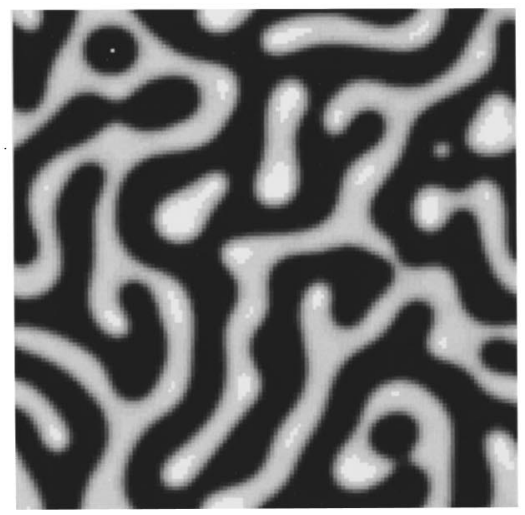

(b)

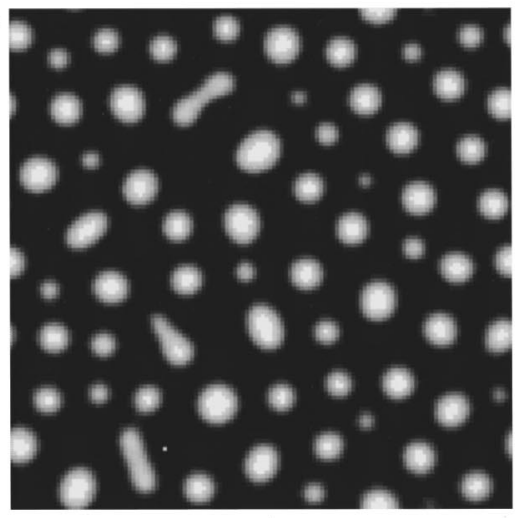

(c)

FIG. 1. Three density patterns of solutions of Eq. (1) at time $t=200$ (or after 10000 time steps; see Appendix A for numerical details) for (a) $\chi_{0}=-0.4$, (b) $\chi_{0}=0.0$, and (c) $\chi_{0}=0.4$; see the text for details.

realizes that this quantity for a single structure grows with its corresponding radius like $t^{1 / 3}$, but, since the number of the structures growing with this time law decreases like $t^{-2 / 3}$, the total length of the interface decreases like $t^{-1 / 3}$. In Fig. 2 we show numerical results, giving in Fig. 2(a) the average size of the structures and in Fig. 2(b) the total amount of interface in the system. The first quantity is calculated by evaluating the circularly averaged correlation function $g(r, t)$,
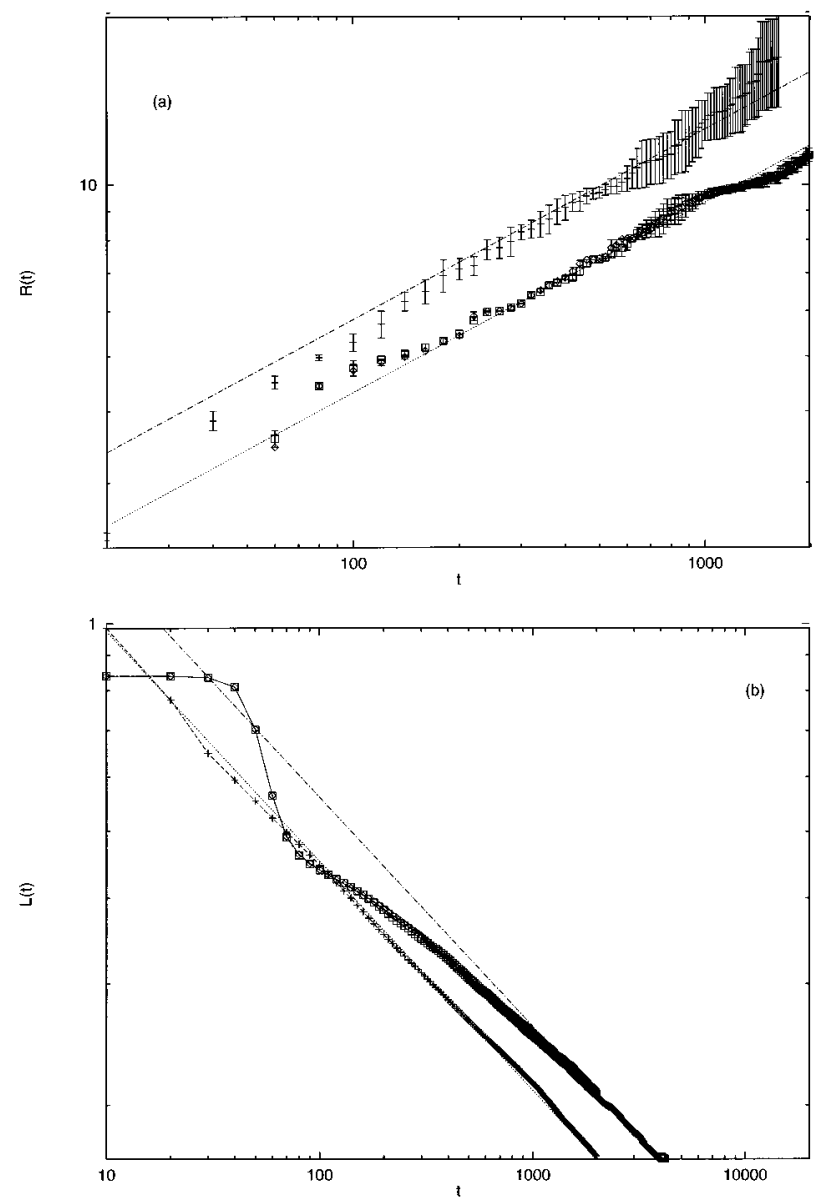

FIG. 2. Characteristic size of structures and total amount of interface as functions of time for three different compositions $\left[\chi_{0}=-0.4\right.$ (diamonds), 0.0 (pluses), and 0.4 (squares) $]$. (a) $\log -\log$ plot of the characteristic size calculated via the circularly averaged correlation function averaged over five realizations (grid size 256) together with two lines (dashed and dotted) that are proportional to $t^{1 / 3}$. (b) log-log plot of the total amount of interface in the system (grid size 512, one realization) together with two lines (dashed and dotted) that are proportional to $t^{-1 / 3}$. Note that for $\chi= \pm 0.4$ the curves coincide.

$$
g(r, t)=\frac{1}{N_{r}} \sum G(\vec{r}, t)
$$

( $N_{r}$ represents the number of points of the corona of radii $r$ and $r+\Delta r$ over which the averaging is taking place) employing the pair correlation function $G(\vec{r}, t)$, which is defined as

$$
G(\vec{r}, t)=\left\langle\frac{1}{N^{2}} \sum_{\vec{r}^{\prime}}\left[\chi\left(\vec{r}+\vec{r}^{\prime}, t\right) \chi\left(\vec{r}^{\prime}, t\right)-\chi_{0}^{2}\right]\right\rangle
$$

(where $N$ is the size of the system). The "characteristic" size $R(t)$ of the structures is now determined by the first zero of the function $g(r, t)$ with respect to $r$. The second of the above mentioned quantities is calculated by evaluating the integral

$$
L(t)=\left\langle\iint d x d y\left(1-\chi^{2}\right)\right\rangle_{\chi}
$$


averaged over different realizations of $\chi$, which obviously has contributions (almost) only from the interface region, i.e., where $\chi$ differs significantly from \pm 1 . While it is known that within the structures the actual value (in two dimensions) of $\chi$ is not exactly \pm 1 because of the curvature, the deviation is small enough to neglect it. In addition to the time laws mentioned, one sees, in particular in Fig. 2(b), that at small times there is not a well-developed phase separation and therefore this integral starts at a value of $1-\chi_{0}^{2}$. Note that the characteristic size of the structures for $\chi_{0}=0.0$ is somewhat larger at every time point and therefore the total amount of interface is somewhat less than for $\chi_{0}= \pm 0.4$. This stems from the fact that we here have lamellar structures and not droplets.

The next step is to assign at every space-time point a diffusion coefficient for the scalar $C$. We chose a very simple coupling between the structured medium and the distribution of diffusion coefficients, namely,

$$
D(\vec{r}, t)=[1-a \chi(\vec{r}, t)] D^{*} .
$$

This quantity will be referred to as the local diffusion coefficient. $D^{*}$ is an input value that together with the parameter $a$ (here $a=0.85$ ) defines the diffusion coefficients in the pure phases. Since the stable (equilibrium) states of Eq. (1) lie at $\chi_{\text {eq }}= \pm 1$ we have $D_{A}=0.15 D^{*}$ and $D_{B}=1.85 D^{*}$. [The reason to choose $a<1$ is to ensure that at every point $D(\vec{r}, t)$ is positive definite; see the discussion below.] The averaged diffusion constant is given by

$$
\langle D(\vec{r}, t)\rangle_{r, \chi}=\left[1-a\langle\chi(\vec{r}, t)\rangle_{r, \chi}\right] D^{*}=\left[1-a \chi_{0}\right] D^{*}
$$

and is therefore constant in time. [The subscripts to the angular brackets intend to show, as above, whether averaging is over space $(r)$, over realizations $(\chi)$, or both.]

This scalar field of diffusion constants is now implemented in the dynamical equation in the following way. Let $\psi(r, t)$ be a passive, i.e., nonreacting, scalar field that describes the density of the substance $C$. Then we have the diffusion equation

$$
\frac{\partial \psi}{\partial t}=\vec{\nabla} \cdot[D(r, t) \vec{\nabla} \psi(r, t)]
$$

which is initialized with a single $\delta$ function usually centered in space. The second moment $\sigma(t)$ and the "diffusion constant" $D(t)$ are calculated by the standard procedure, namely,

$$
\begin{aligned}
\sigma(t)= & \left\langle(\Delta r)^{2}\right\rangle_{r, \chi}(t)=\left\langle r^{2}\right\rangle_{r, \chi}-\langle r\rangle_{r, \chi}^{2} \\
= & \iint d x d y\left(x^{2}+y^{2}\right)\langle\psi\rangle_{\chi}-\left(\iint d x d y x\langle\psi\rangle_{\chi}\right)^{2} \\
& -\left(\iint d x d y y\langle\psi\rangle_{\chi}\right)^{2} .
\end{aligned}
$$

This average mean-square displacement is the key quantity presented in the work. As is very well known, for homogeneous media this quantity is proportional to time, which is why one most often uses instead the number

$$
D(t)=\frac{\sigma(t)}{4 t}
$$

in two dimensions. For the sake of simplicity we will also call this quantity the diffusion constant. For every homogeneous medium with diffusion coefficient $D^{*}$ (i.e., for example, if we set the above parameter $a$ equal to zero, thus suppressing the coupling between the diffusion and the evolving pattern) this yields a constant value of $D^{*}$.

The temporal development of $\sigma(t)$ is subject to quite a number of parameters. From the Cahn-Hilliard equation stems the first of those parameters: the composition $\chi_{0}$. Second, one has the parameters $a, D^{*}$, and $\langle D\rangle$. (Of these four parameters, only three are independent, of course.) Finally, one may consider freezing the temporal development of the Cahn-Hilliard equation or likewise retard the start of the diffusion equation, thus letting the pattern evolve at first until structures are visible. Our main point addressed here will be whether we reach a constant $D(t)$ after a finite time.

To gain some understanding in the process and furthermore to get an idea of the key quantities involved, we derived in Appendix A an expression for $D(t)$ that reads

$$
\begin{aligned}
D(t)= & D^{*}-\frac{a D^{*}}{t} \int_{0}^{t} d t^{\prime} \iint d x d y\langle\chi \psi\rangle_{\chi} \\
& -\frac{a D^{*}}{2 t} \int_{0}^{t} d t^{\prime} \iint d x d y\left[\left(x-\langle x\rangle_{r, \chi}\right)\left\langle\frac{\partial \chi}{\partial x} \psi\right\rangle_{\chi}\right. \\
& \left.+\left(y-\langle y\rangle_{r, \chi}\right)\left\langle\frac{\partial \chi}{\partial x} \psi\right\rangle_{\chi}\right],
\end{aligned}
$$

which, according to our simulations also mentioned in Appendix A, can be extremely well approximated by

$$
\begin{aligned}
D(t)= & D^{*}\left(1-a \chi_{0}\right)-\frac{a D^{*}}{2 t} \int_{0}^{t} d t^{\prime} \\
& \times \iint d x d y\left\langle\left(x \frac{\partial \chi}{\partial x}+y \frac{\partial \chi}{\partial y}\right) \psi\right\rangle_{\chi} \\
\equiv & \langle D\rangle_{r, \chi}-\int_{0}^{t} \delta D\left(t^{\prime}\right) d t^{\prime} .
\end{aligned}
$$

The first part of this equation is exactly the average diffusion constant $\langle D\rangle$, which is therefore constant in time. The time dependence of $D(t)$, apart from short-time effects, therefore has to come from the second part of this equation, which we will call $\delta D(t)$.

The time dependence of $D(t)$ is shown in Fig. 3. All three simulation results for $\chi_{0}=-0.4,0.0$, and 0.4 depart from the statistically averaged value of $\langle D\rangle=0.5$ and evolve then decreasing at a rate depending on $\chi_{0}$. This indicates a growth of $\sigma(t)$ that is less than linear for the time of the simulation. (This corresponds to at least $5 \times 10^{5}$ time steps; see Appendix B for numerical details. In the case of $\chi_{0}=0.4$ the simulation has even been carried out up to time $t=20000$ without changing the results shown in the Fig. 3.)

Clearly, this "sublinear" growth of $\sigma(t)$ depends not only on the dependence on $\chi_{0}$ shown, but also on the cou- 

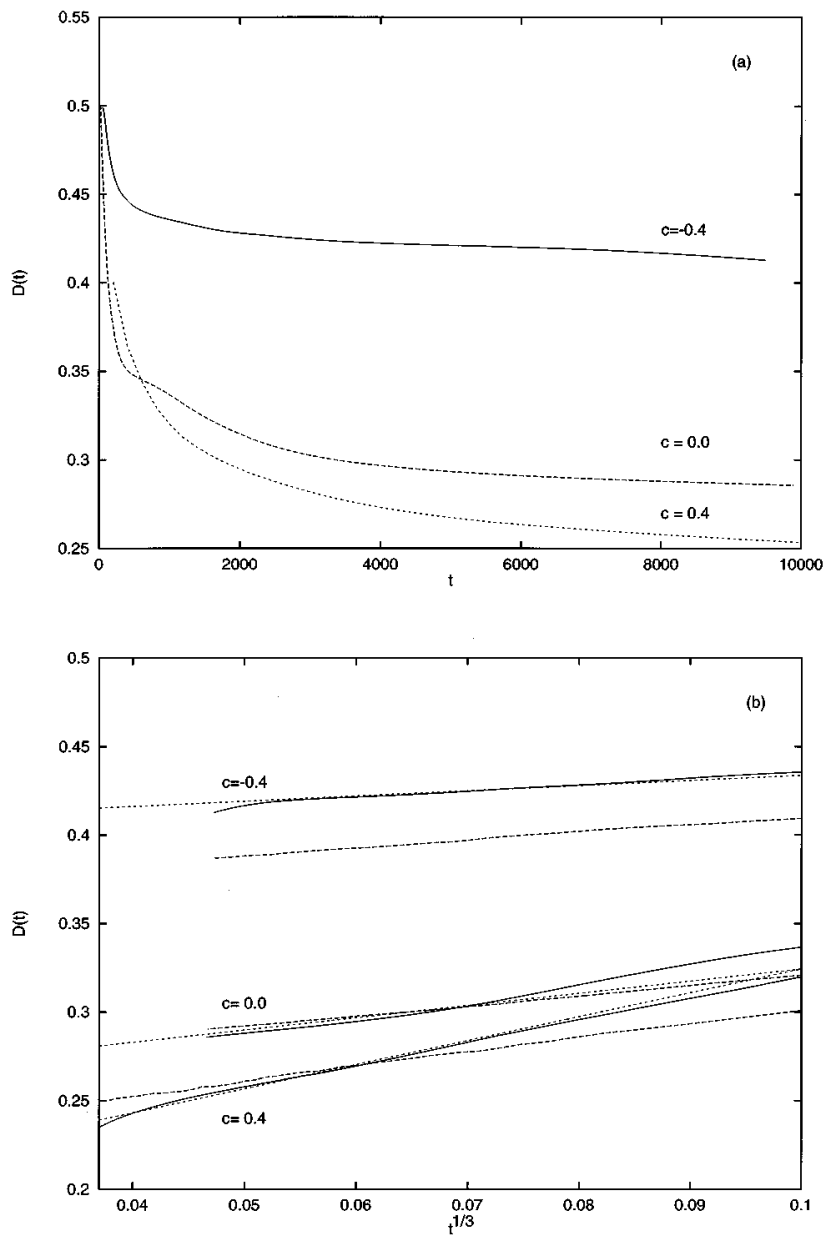

FIG. 3. "Diffusion constant" $D(t)$ as a function of time for three different compositions $\left[\chi_{0}=-0.4\left(D^{*}=0.37\right), 0.0\right.$ $\left(D^{*}=0.50\right)$, and $\left.0.4\left(D^{*}=0.76\right)\right]$. (a) Data in a conventional linear plot (solid line for $\chi_{0}=-0.4$, long-dashed line for $\chi_{0}=0.0$, and short-dashed line for $\chi_{0}=0.4$ ). (b) Data plotted against $t^{-1 / 3}$ (solid lines). Shown also are fits to $D_{\infty}^{\text {fit }}+b t^{-1 / 3}$ (short-dashed line) and the results of corresponding EMA calculations (long-dashed line). The composition $\chi_{0}$ is by $c$. Note that neither the simulation nor the EMA reaches a constant value, although the simulation time is very large. For $\chi_{0}=-0.4$ finite-size effects are visible for large times. (The grid size is 512.)

pling parameter $a$. The deviation of the usual linear time law will be greatest when $a$ approaches 1 and negligible when $a$ goes to zero. The case of $a=1$ is not accessible to us since at the points where $\chi$ exceeds its equilibrium value of +1 the local diffusion coefficient defined by Eq. (3) would not be positive (semi)definite. Nevertheless, this case has already been treated for a temporarily static medium by different methods in the literature, mostly in the framework of percolation theory [10-13] and using random-walk techniques. One immediately realizes that the value $a=1$ (or $D_{A}=0$ ) is a rather special value yielding results not only qualitatively different from the case treated here. The first important difference is the existence of a percolation threshold, which does not exist in the problem posed here. We do observe around the value of $\chi_{0}= \pm 0.18$ (which corresponds to the percolation threshold of a square lattice) a structural phase transition from lamellar to droplet configurations, but in our case these values of $\chi_{0}$ are in no way extraordinary. Quite on the contrary, in the treatment of diffusion in the presence of obstacles (i.e., $D_{A}=0$ ), where around this value an infinite and fractal cluster is formed, this leads to sublinear diffusion

$$
\sigma(t) \sim t^{2 / d_{w}}
$$

with $d_{w}>2$. Its cause is the fractal nature of the cluster formed. Above the percolation threshold, long-range diffusion is impossible, whereas in our treatment diffusion always takes place. Owing to these differences in the ansatz, it results that quite different concepts are of importance and therefore quite different results can be obtained when leaving the rather special value of $D_{A}=0$. Saxton treated diffusion in the presence of obstacles in a series of papers (see [13] and references therein) for a wide range of different parameters, among those, e.g., the obstacle size. He found in his static simulations, keeping the relative area of obstacles-medium constant, which in our language means keeping $\chi_{0}$ constant, that $D(t)$ quite generally increases with the obstacle size, indicating that in the limit of an infinite system with infinite obstacle size $\sigma(t)$ would simply grow linearly with slope $4\langle D\rangle$. Saxton attributes this to the fact that for growing obstacle size there are fewer obstacles in the system and therefore diffusion is less hindered. Looking now at Fig. 3 one finds quite the contrary. In our dynamical treatment the "obstacle" size grows with time (like $t^{1 / 3}$ ) and their number decreases with $t^{-2 / 3}$, keeping their relative area constant just as in the case of the static treatment by Saxton. Nevertheless, in our case $D(t)$ evolves ever decreasing. This small but illustrative example shows already (besides other considerations, such as, for example, the missing percolation threshold and the even so missing fractal nature of our "clusters"') that one has to consider the cases $a=1$ and $a \neq 1$ as rather different classes of a seemingly similar problem.

\section{ROLE OF THE INTERFACE}

Looking closely at the integral representing $\delta D(t)$ in Eq. (8), one sees that the function to be integrated spatially gets its contributions almost exclusively from the regions of the interface, where the derivatives $(\partial \chi / \partial x$ and $\partial \chi / \partial y)$ are significantly different from zero. This means that the interface and its temporal development will play a decisive role in $\sigma(t)$. As the interface in our treatment develops dynamically, one has to expect a time dependence of $D(t)$, at least until the importance of the interface is overrun by the averaging process taking place due to the growth of the distribution $\psi$. Let us note in passing that the existence of an interface marks another strong difference from the case considered by Saxton and others. In his treatment obstacles are regions excluded from the available positions of the particles and therefore there does not exist an interface in the sense of Eq. (8).

The physical cause behind the importance of the interface is that the regions of low diffusivity act as reservoirs. Here the diffusivity is so small that it takes a very long time for the matter to diffuse through these regions. Therefore, even at longer times, a good part of the dispersed matter will be collected in exactly these regions, thus giving rise to an ever decreasing $D(t)$. Moreover, these regions have their own dynamics, which gives a process that will only asymptoti- 


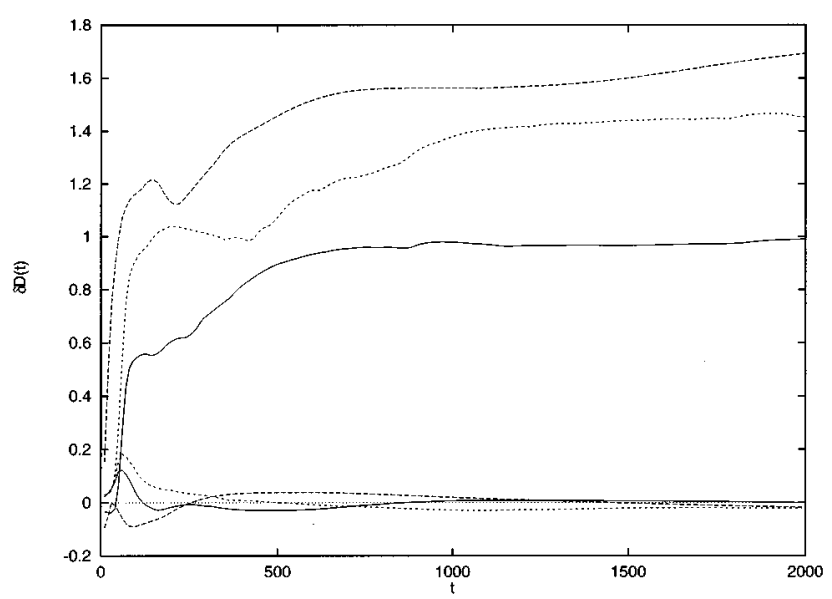

FIG. 4. Integral $\iint d x d y\left(x \chi^{\prime}+y \chi^{\prime}\right) \psi$ for three different choices of $\chi_{0}$ corresponding to those of Fig. 3. The three curves in the upper part of the figure correspond to calculations with $a=0.85 \quad(\chi=-0.4$, solid line, $\chi=0.0$, long-dashed line; and $\chi_{0}=0.4$, short-dashed line), while the other three give results for $a=0 \quad\left(\chi=-0.4\right.$; solid line, $\chi=0.0$, long-dashed line; and $\chi_{0}=0.4$ short-dashed line). The latter choice ensures that the distribution entering the above integral is of Gaussian shape with a fixed halfwidth. Here $D^{*}$ was kept constant at a value of 0.5 . As one observes there are only short-time effects, decaying rapidly to zero, while this is not true for the choice $a=0.85$. See the text for a discussion.

cally reach a finally constant value for $D(t)$. This final value reached is a subject that deserves a more thorough treatment, which is why we will postpone this until later. To somewhat substantiate the above general comment let us show results of two different kinds of calculations that show the importance of the "reservoir effect'" and its decisive influence on the overall temporal development of $D(t)$.

Let us begin by eliminating altogether a possible reservoir effect. To achieve this we calculate the function $\delta D(t)$ appearing in Eq. (8), only now with $a=0$, and compare this to our usual choice $a=0.85$. Clearly, when setting $a=0$ this is equivalent to the homogeneous case and therefore the distribution of the scalar will have the usual Gaussian form

$$
\psi=\frac{e^{-\left(x^{2}+y^{2}\right) / 4 D^{*} t}}{4 \pi D^{*} t}
$$

(The fact that here $D^{*}$ appears, and not a different value or even a fit parameter, is solely of quantitative importance.) In other words, we are presupposing a solution that is completely uncoupled from the surface and therefore exhibits absolutely no reservoir effects. Results for these calculations in comparison with those for $a=0.85$ are collected in Fig. 4. As one can easily observe, the behavior is very different. Most important of all, in the long-time limit $\left.\delta D\right|_{a=0}$ tends to zero, which is absolutely not the case for the "exact", $\delta D(t)$. Additionally, in the short-time limit, we find even significantly negative values for $\left.\delta D\right|_{a=0}$, but not for $\delta D(t)$. It can be shown by simulations and general considerations that the latter is only a short-time effect and therefore of no further importance for the discussion here. The most important result here is that the Gaussian approxima-

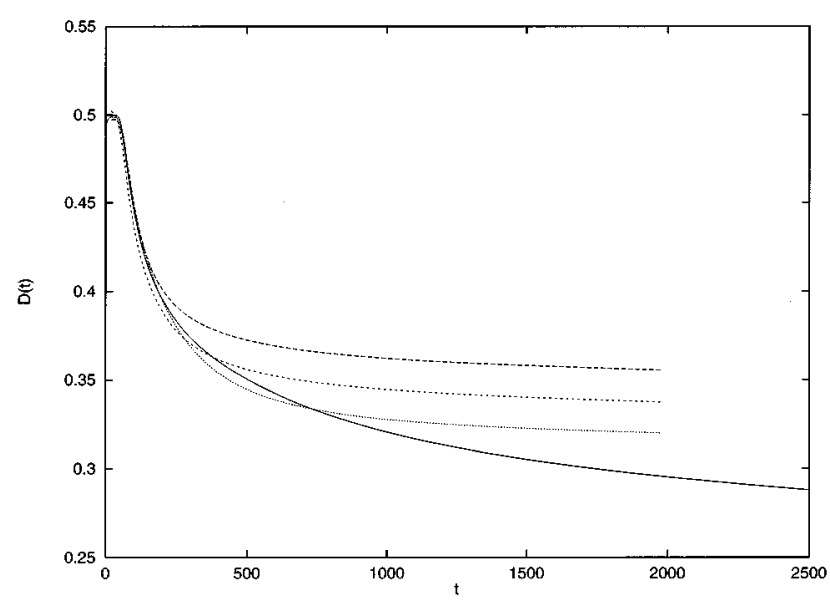

FIG. 5. $D(t)$ for four situations. The solid line represents simulation results (averaged over five realizations) with sustained CahnHilliard dynamics. For the other graphs the dynamics of the phase separation were stopped at $t_{F}=100,200$, and 300 (long-dashed, short-dashed, and dotted lines, respectively). Obviously, $D(t)$ for the frozen dynamics decays much faster to an asymptotic value, which depends on the total amount of interface present at time $t_{F}$. (The grid size is $256, \chi_{0}=0.4$, and $D^{*}=0.757576$.)

tion is incompatible with the results obtained for $a=0.85$, since it yields a completely different time development. This means that disregarding the reservoir effect is not adequate for treating the problem we pose here. Keeping in mind that the Gaussian form for $\psi$ is equivalent to effective diffusion with some constant value for $D(t)$ one can already see here that the existence of low diffusivity regions prolongs considerably the usually fast transitory regime to the said effective diffusional process.

To see in which manner we have to expect to reach the final diffusional regime, we have also solved the diffusion equation (4) on "frozen" configurations of the Cahn-Hilliard equations, i.e., we initialize both the Cahn-Hilliard equation and the diffusion equation at the same time. Then we solve both equations up to some time $t_{F}$ (where $F$ stands for "freezing'), where we stop the temporal development of $\chi$ and retain that of $\psi$. In this way, the only time dependence in $\delta D$ is that of $\psi$. Results of this type of calculation are shown in Fig. 5 for $t_{F}=100,200,300$. The freezing times were chosen to be small in order that the structures remain small themselves, but large enough to ensure that $\chi$ has already reached its equilibrium values. The situation is now as follows: We have retained the "reservoir effect,"' but neglected that the "reservoirs" themselves have a temporal development. Although the data presented in Fig. 5 do not explicitly show a new stationary value for $D(t)$, quite obviously we will reach a new purely diffusive regime much faster than with sustained growth of the structures. Being the slower process, the overall $D(t)$ has to depend therefore quite directly on the time development of the total length of the interface, i.e., we may now assume that for very large times, where the temporal dependence of $D(t)$ due to the spreading of $\psi$ is negligible, $D(t)$ has to go like the length of the interface reaching at infinite times a final value still to be determined. This argumentation is restricted obviously to the case where the time scales of the spreading of $\psi$ and the 
TABLE I. Estimation of $D_{\infty}$ for a constant value of $\langle D\rangle=\left(1-a \chi_{0}\right) D^{*}=0.5$. $\left(a=0.85\right.$.) $D_{A}$ and $D_{B}$ signify the values of the diffusion coefficient for the pure phase $A(\chi=+1)$ or $B(\chi=-1)$, respectively. $D_{\infty}^{\mathrm{EMA}, L}$ is the approximation to $D_{\infty}$ within the effective-medium ansatz for a finite system. It is numerically calculated using condition (9) under the assumption that the finally reached distribution of $\chi$ is a single circular structure (with interface). $D_{\infty}^{\mathrm{EMA}}$ is calculated using (10) and therefore is a result for an infinite system with a completely sharp interface. $D_{\infty}^{\text {fit }}$ is obtained by fitting the simulation data to $D_{\infty}^{\mathrm{fit}}+b t^{-1 / 3}$ and the errors estimated by varying $D_{\infty}^{\text {fit }}$ (adjusting after that the parameter $b$ ) until the sum of squares of residuals is double its optimal value. See the text for further details.

\begin{tabular}{lcccccc}
\hline \hline$\chi_{0}$ & $D^{*}$ & $D_{A}$ & $D_{B}$ & $D_{\infty}^{\text {EMA }, L}$ & $D_{\infty}^{\text {EMA }}$ & $D_{\infty}^{\text {fit }}$ \\
\hline 0.4 & 0.7576 & 0.1136 & 1.4015 & 0.2281 & 0.2174 & $0.193 \pm 0.005$ \\
0.0 & 0.5000 & 0.0750 & 0.9250 & 0.2776 & 0.2634 & $0.256 \pm 0.005$ \\
-0.4 & 0.3731 & 0.0560 & 0.6903 & 0.3699 & 0.3608 & $0.40 \pm 0.01$ \\
\hline \hline
\end{tabular}

development of the interface length $L(t)$ are well separated and does not apply, e.g., to the case of frozen configurations, where the temporal development of $D(t)$ is not at all governed by the (overmore constant) $L(t)$. But, as we are interested here in the dynamical effects of the phase separating medium and have considered the "frozen" case only in order to find out the different time scales of the problem, this is not a restriction for our treatment.

In short terms, we have to introduce two concepts (foreign to the treatment where $D_{A}=0$ ), namely, that of the reservoirs and that of the interface in order to interpret consistently the results obtained. The reservoir effect is in our view the physical cause behind the prolonged sublinear regime of $\sigma(t)$ and the dynamically evolving interface length an adequate measure for it. These reservoirs or "sinks" prevent $\psi$ from reaching a Gaussian shape with some linear variance $\left\langle\Delta r^{2}\right\rangle$ for finite times because of their own dynamics, which thus dominates the process. We included therefore, in Fig. 3(b), fits to

$$
D(t)=D_{\infty}^{\mathrm{fit}}+b t^{-1 / 3}
$$

with $D_{\infty}^{\text {fit }}$ (see also Table I) and $b$ being free parameters and $t^{-1 / 3}$ the time development of the total interface length. Of the many possible manners of representation we chose a plot of the data of Fig. 3(a) versus $t^{-1 / 3}$, where the test functions represent straight lines. Note that in this plot time grows from right to left. Therefore special attention has to be paid to the left part of the plot. Finite-size effects, clearly visible for the case of $\chi_{0}=-0.4$ in Fig. 3(a), plotted by the change of slope at higher times, are somewhat obscured in Fig. 3(b) due to the small portion of the axis representing those times. The initial disagreement of the fit in the case of $\chi_{0}=0.0$ up to $t^{-1 / 3}=0.07$ can be attributed to the somewhat nonsmooth initial behavior in this case [see Fig. 3(a)] due to the fact that the data shown are for one realization only; note also that $t^{-1 / 3}=0.07$ corresponds approximately to $t=3000$. (The simple reason for using only one realization is the computer time needed; each graph needs approximately $15 \mathrm{~d}$ of pure computer time on a Silicon Graphics Indigo [2].) Apart from these considerations, one can see that the numerical data are represented reasonably well by the above test function.

\section{THE ASYMPTOTIC VALUE $D_{\infty}$}

Now let us turn to the reached value asymptotically for infinite times. Except for fitting the data to a presumed test function, we will try to find an approximation to this value by using an alternative method based on an effectivemedium ansatz.

Based on a very simple argument using a kind of effective-medium approximation (EMA) one can derive a condition (see, e.g., [14])

$$
\left\langle\frac{2 D_{\infty}^{\mathrm{EMA}}}{D_{\infty}^{\mathrm{EMA}}+D(\vec{r})}\right\rangle_{r, \chi}^{!}=1,
$$

which after solving gives an approximation to the asymptotically reached diffusion constant for a given distribution of local diffusion coefficients. Let us note in passing that this average has three contributions: one from the $A$ zone, one from the $B$ zone, and, finally, one from the interface region. Here again the crucial role of the interface is stressed since the first two contributions have extremely little time dependence.

We have employed this approximation in two different manners. First, instead of treating our problem within a timedependent EMA framework, we evaluated the above expression at different times $t_{1}<t_{2}<\cdots$ during the simulation, thereby obtaining the asymptotic $D_{t_{1}}^{\mathrm{EMA}}, D_{t_{2}}^{\mathrm{EMA}}, \ldots$ for the spatially inhomogeneous but stationary case. Then we define a curve joining this set of values, thus yielding an approximation to $D(t)$. We have calculated this kind of "adiabatic", effective-medium approximation; the results are presented together with the simulation results in Fig. 3(b). For the moment this serves one sole purpose: to show that this kind of approximation gives very reasonable results. In fact, we found the error (assuming the simulation results to be exact) to be generally less than $10 \%$. Note, though, that the temporal development is slightly different.

Second, and of more importance here, we used this approximation in its original sense, namely, to find an approximation to $D_{\infty}$. This is done using the following argumentations, i.e., one considering a finite system and one considering an infinite system.

As mentioned, in our phase-separating problem, the number of structured domains decreases with time. That means that at an infinite time we are left with one single droplet of $A$ immersed in $B$ (or vice versa), with relative area given by $\left(1 \pm \chi_{0}\right) / 2$. Using now the equilibrium solution of the CahnHilliard equation (the interface goes like a hyperbolic tangent) we are able to numerically solve the above condition (9), i.e., we solve this equation on a surface with a single circular structure with the radial dependence being $\tanh [(r-R) / \sqrt{2}]$, where $R$ is the $\chi_{0}$-dependent size of the 
droplet. This should give us an approximation to the asymptotic value of $D(t)$ for a finite system.

In the limit of an infinite system we can even go one step further. If the system is infinite the relative width of interface present goes to zero. Assuming therefore that there is a completely sharp interface and that the local diffusion coefficient is simply that of the corresponding pure phase, we can say that

$$
1 \stackrel{!}{=} \frac{1+\chi_{0}}{2} \frac{2 D_{\infty}^{\mathrm{EMA}}}{D_{\infty}^{\mathrm{EMA}}+(1-a) D^{*}}+\frac{1-\chi_{0}}{2} \frac{2 D_{\infty}^{\mathrm{EMA}}}{D_{\infty}^{\mathrm{EMA}}+(1+a) D^{*}},
$$

where $\left(1+\chi_{0}\right) / 2\left[\left(1-\chi_{0}\right) / 2\right]$ is again the fraction of the area occupied by phase $A[B]$ where the diffusion coefficient is $(1-a) D^{*}\left[(1+a) D^{*}\right]$. This is a simple quadratic equation and has the physical solution

$$
D_{\infty}^{\mathrm{EMA}}=D^{*}\left[-a \chi_{0}+\sqrt{1-a^{2}\left(1-\chi_{0}^{2}\right)}\right],
$$

which can now be used to calculate the wanted $D_{\infty}^{\mathrm{EMA}}$. (Note that for the case of $\chi_{0}=0.0$ this yields the classical result $D_{\infty}^{\mathrm{EMA}}=\sqrt{D_{A} D_{B}}$.)

The value of calculating $D_{\infty}^{\mathrm{EMA}}$ in these two different manners lies in that we are now able to countercheck the results thus obtained. Clearly, both have to yield values similar to those of the finite system being slightly larger than the others.

In Table I we present the data concerning this section. As can be seen from the table, for very large times and a single droplet the influence of the interface is rather small, so that the rather simplistic ansatz that led to Eq. (10) gives very reasonable results.

These values can be compared to the ones obtained before by fitting the simulation data to $D_{\infty}^{\mathrm{fit}}+b t^{-1 / 3}$. The values $D_{\infty}^{\mathrm{fit}}$ are listed in the last column of Table I. The errors given there for these quantities have been evaluated by varying $D_{\infty}^{\text {fit }}$ (adjusting the parameter $b$ every time) until the sum of squares of residuals was double its optimal value using the "best" parameter set. The values obtained from these different procedures agree reasonably and best for $\chi_{0}=0.0$, which agrees with the fact that the EMA is a good approximation either for small differences of concentrations of $A$ and $B$ or for small concentrations of $A$ in $B$ (or vice versa). The overall error, assuming again the simulation results to be exact, is, like in the case of the "adiabatically" used EMA, about $10 \%$.

\section{SUMMARY AND DISCUSSION}

In this paper we treated diffusion in a special inhomogeneous medium, namely, in a phase separating binary alloy, whose temporal development is determined by the CahnHilliard equation. With the exception of few calculations we considered the following situation. At time $t=0$ we insert a "droplet" of a passive substance $C$ in a medium that has not yet started its phase separation. The medium consists of two substances $A$ and $B$, in which $C$ diffuses at a different rate, neither of which vanishes. The respective diffusion coefficients for the pure phases are called $D_{A}$ and $D_{B}$. After having thus prepared the system, we start the time evolution and measure the spatially averaged diffusion "constant" $D(t)$ of the substance $C$ in the medium by determining the variance of its distribution function.

We found that $D(t)$, independent of the initial composition of $A$ and $B$, decreases with time. What is more, for very long times it decreases like $t^{-1 / 3}$, which is an extremely slow process. A nonzero constant value for $D(t)$ is expected to be reached at infinite times, but this is (a) an asymptotic process that takes (b) a very long time even to reach only an approximately constant value. As can be seen from the simulation results presented in Fig. 3, after $5 \times 10^{5}$ time steps or at time $t=10000$ we are still far away from the said constant value $D_{\infty}$; compare also Table I. With respect to the time law found, therefore, one might say that diffusion, at least in this special medium, is sublinear, had the latter term not been reserved for $D_{\infty}=0$.

The reason for the deviation from the typical linear dependence of $\sigma(t)$ has been localized in relation to Eq. (8). Here one easily recognizes that the interface and its temporal development introduce a new time dependence. Besides short-time effects, which more specifically are here (a) the initial deviation of the length of the interface from $t^{-1 / 3}$ and (b) effects due to the initially comparable size of the structures (which grow like $t^{1 / 3}$ ) and of the distribution $\psi$ of the scalar $C$ (which grows a little less than linear), there is a long-time tail proportional to the total length of the interface. This means that finally $D(t)$ goes like $t^{-1 / 3}$ to a constant value. We have fitted the simulation data therefore to a test function

$$
D(t) \approx D_{\infty}^{\mathrm{fit}}+b t^{-1 / 3}
$$

with very reasonable success (see also Fig. 3). The value $D_{\infty}^{\text {fit }}$ gives an estimation to the asymptotic value reached for infinite times. (Let us insist once more that the above fit is justified only in the case of a fully dynamical system. For "frozen" configurations, where one stops the temporal development of the Cahn-Hilliard equation, this ansatz is not justified. But since it is quite clear that without the slow dynamics of the phase separation we would find after a short transient an effective diffusion, this case seems not to be sufficiently interesting.)

In order to estimate the latter we also used an approximative treatment based on an effective-medium ansatz, which in this case led to Eq. (9). Using now the simple argument that at infinite time we remain with only one single droplet and using the equilibrium solution of the Cahn-Hilliard equation, we could numerically solve the above condition for this finite system. If, on the other hand, we consider the system to be infinitely large, then at $t=\infty$ the relative amount of the interface is negligible and Eq. (9) can be solved analytically. The values obtained in both ways are very well comparable with those of the finite system being slightly larger, as one has to expect, and in turn compare well with those obtained by the above-mentioned fit with a deviation of about $10 \%$.

Keeping in mind that the cause of the nonlinear time dependence of $\sigma(t)$ is the sole existence of reservoirs with an interface, which develop in time on a slower time scale than the spreading of $\psi$, we expect that the behavior found in this special treatment is very well generalizable to other dynamics of inhomogeneous media. 


\section{ACKNOWLEDGMENTS}

H. L. would like to thank F.S. for having made the stay in his group possible. We would also like to thank Dr. I. Sokolov for discussions about the effective-medium approximation. This work was supported by the Generalitat de Catalunya under the CIRIT-PIEC program and by the Deutsche Forschungsgemeinschaft.

\section{APPENDIX A: DERIVATION OF EQ. (7)}

We follow here the approach used earlier in Ref. [15]. The most important step is to calculate the mean-square displacement via its time derivative

$$
\frac{\partial\left\langle(\Delta r)^{2}\right\rangle_{r, \chi}}{\partial t}=\int d \vec{r}(\Delta \vec{r})^{2} \frac{\partial\langle\psi\rangle_{\chi}}{\partial t} .
$$

Since now $D(\vec{r}, t)=D^{*}[1-a \chi(\vec{r}, t)]$ one finds for the latter derivative

$$
\begin{aligned}
\frac{\partial}{\partial t}\langle\psi\rangle_{\chi} & =\langle\vec{\nabla} \cdot[D(\vec{r}, t) \vec{\nabla} \psi]\rangle_{\chi} \\
& =\left\langle\vec{\nabla} D(\vec{r}, t) \cdot \vec{\nabla} \psi+D(\vec{r}, t) \nabla^{2} \psi\right\rangle_{\chi} \\
& =D^{*}\left\{\left\langle\nabla^{2} \psi\right\rangle_{\chi}-a\langle(\chi \vec{\nabla}+\vec{\nabla} \chi) \cdot \vec{\nabla} \psi\rangle_{\chi}\right\} .
\end{aligned}
$$

Keeping in mind that

$$
\frac{\partial}{\partial t}\left\langle(\Delta r)^{2}\right\rangle_{r, \chi}=\frac{\partial}{\partial t}\left(\left\langle r^{2}\right\rangle_{r, \chi}-\langle r\rangle_{r, \chi}^{2}\right)
$$

and that furthermore

$$
\begin{aligned}
\frac{\partial}{\partial t}\langle r\rangle_{r, \chi}^{2} & =2 \int d \vec{r} \vec{r} \frac{\partial}{\partial t}\langle\psi\rangle_{\chi} \cdot \int d \vec{r} \vec{r}\langle\psi\rangle_{\chi} \\
& =2 \frac{\partial\langle\vec{r}\rangle_{r, \chi}}{\partial t}\langle\vec{r}\rangle_{r, \chi},
\end{aligned}
$$

one deduces for the average of the mean displacement (or rather its temporal derivative) using partial integration

$$
\frac{\partial}{\partial t}\langle r\rangle_{r, \chi}=a D^{*}\left(\int d \vec{r}\langle\chi \vec{\nabla} \psi\rangle_{\chi}\right) .
$$

Let us turn now to the square part. Obviously the first term on the right-hand side of Eq. (A1) is the original term for diffusion in homogeneous media. Therefore we can immediately give the solution to this part as $4 D^{*}$. We get now

$$
\begin{aligned}
\frac{\partial}{\partial t}\left\langle r^{2}\right\rangle_{r, \chi}= & 4 D^{*}-a D^{*} \iint d x d y\left[x^{2}\left\langle\chi \nabla^{2} \psi\right\rangle_{\chi}\right. \\
& \left.+x^{2}\langle\vec{\nabla} \chi \vec{\nabla} \psi\rangle_{\chi}+(\text { terms in } y)\right] .
\end{aligned}
$$

Contributions of mixed terms (e.g., $x^{2} \partial^{2} / \partial y^{2}$ ), as usual, do not give any contribution and therefore $\vec{\nabla}$ in the above equation can be substituted by $\partial / \partial x$ (or $\partial / \partial y$ in the $y$ terms, naturally). Using now again partial integration of the first term of the above equation (the remainder $\left[x^{2}\langle\chi \partial \psi / \partial x\rangle\right]$ vanishes because of periodic boundary conditions) one finds

$$
\begin{aligned}
\frac{\partial}{\partial t}\left\langle r^{2}\right\rangle_{r, \chi}= & 4 D^{*}+2 a D^{*} \iint d x d y x\left\langle\chi \frac{\partial}{\partial x} \psi\right\rangle_{\chi} \\
& +2 a D^{P *} \iint d x d y y\left\langle\chi \frac{\partial}{\partial y} \psi\right\rangle_{\chi} \\
= & 4 D^{*}-2 a D^{*} \iint d x d y\left[2\langle\chi \psi\rangle_{\chi}\right. \\
& \left.+x\left\langle\frac{\partial \chi}{\partial x} \psi\right\rangle_{\chi}+y\left\langle\frac{\partial \chi}{\partial y} \psi\right\rangle_{\chi}\right] .
\end{aligned}
$$

Now putting everything together, we find

$$
\begin{aligned}
\frac{\partial}{\partial t}\left\langle(\Delta r)^{2}\right\rangle_{r, \chi}= & 4 D^{*}-2 a D^{*} \iint d x d y\left[2\langle\chi \psi\rangle_{\chi}\right. \\
& +\left(x-\langle x\rangle_{r, \chi}\right)\left\langle\frac{\partial \chi}{\partial x} \psi\right\rangle_{\chi} \\
& \left.+\left(y-\langle y\rangle_{r, \chi}\right)\left\langle\frac{\partial \chi}{\partial y} \psi\right\rangle_{\chi}\right],
\end{aligned}
$$

or, integrating over time,

$$
\begin{aligned}
\sigma(t)= & 4 D^{*} t-2 a D^{*} \int_{0}^{t} d t^{\prime} \iint d x d y\left[2\langle\chi \psi\rangle_{\chi}\right. \\
& \left.+\left(x-\langle x\rangle_{r, \chi}\right)\left\langle\frac{\partial \chi}{\partial x} \psi\right\rangle_{\chi}\left(y-\langle y\rangle_{r, \chi}\right)\left\langle\frac{\partial \chi}{\partial y} \psi\right\rangle_{\chi}\right] .
\end{aligned}
$$

Let us now discuss this equation. The very first term represents naturally the "normal" or homogeneous diffusion. The second term (the first below the integral) represents an average of correlations between the inhomogeneous phase and the density of the diffusing scalar. Clearly, starting, e.g., from a well-developed pattern with already large structures, this term might display a complicated time dependence. Starting instead, which is the case we are most interested in, from a random distribution where therefore the initial droplet of $\psi$ is already of the same order of size as the "structures,", this term will give a linear contribution from the very beginning with a slope of exactly $\chi_{0}$. We checked this numerically and found it to be true to very good precision.The third term at last contains the average position, which as usual is not significantly time dependent. Therefore we reach the final result

$$
\begin{aligned}
\sigma(t)= & 4 D^{*}\left[\left(1-a \chi_{0}\right) t-\frac{a}{2} \int_{0}^{t} d t^{\prime}\right. \\
& \left.\times \iint d x d y x\left\langle\frac{\partial \chi}{\partial x} \psi\right\rangle_{\chi}+y\left\langle\frac{\partial \chi}{\partial y} \psi\right\rangle_{\chi}\right],
\end{aligned}
$$

which can also be rewritten as 


$$
\begin{aligned}
\sigma(t)= & 4\langle D\rangle_{r, \chi} t-2 a D^{*} \int_{0}^{t} d t^{\prime} \\
& \times \iint d x d y\left[x\left\langle\frac{\partial \chi}{\partial x} \psi\right\rangle_{\chi}+y\left\langle\frac{\partial \chi}{\partial y} \psi\right\rangle_{\chi}\right] .
\end{aligned}
$$

\section{APPENDIX B: NUMERICAL DETAILS}

For all our calculations we have used a square grid. Discretization was made uniformly using a space grid of usually $\Delta=1$ (for checking purposes we also used $\Delta=0.5$, but with this choice the appropriate time step $\Delta t$ to ensure numerical stability is so small that it forbids using this as a standard). Time has also been discretized uniformly with $\Delta t$ being 0.020 or 0.025 . The grid size $N$ was 128,256 , or 512 points, depending on the simulation time.

The norm of $\psi\left[=\Sigma_{i j} \psi\left(x_{i}, y_{j}, t\right)\right]$ has been monitored and found to be constant to machine precision. The same was true for the norm of $\chi$. Isotropy was also checked and found to be preserved statistically.

In order to ensure norm conservation it is of major importance to use the correct discretization scheme. In particular the derivative of the product $D \nabla \psi$ deserves some attention. We found that the following discretizations of Eqs. (1) and
(4), respectively, have the desired properties:

$$
\begin{aligned}
\chi_{i, j}(t+\Delta t)= & \chi_{i, j}-\frac{\Delta t}{\Delta^{2}}\left[J_{i+1, j}+J_{i-1, j}+J_{i, j+1}\right. \\
& \left.+J_{i, j-1}-4 J_{i, j}\right]
\end{aligned}
$$

where

$$
J_{i, j}=\left(\frac{4}{N^{2}}-1+\chi_{i, j}^{2}\right)-\frac{1}{\Delta^{2}}\left(\chi_{i+1, j}+\chi_{i-1, j}+\chi_{i, j+1}+\chi_{i, j-1}\right)
$$

and

$$
\begin{aligned}
\psi_{i, j}(t+\Delta t)= & \psi_{i, j}+\frac{\Delta t}{\Delta^{2}} D_{i j}\left(\psi_{i+1, j}+\psi_{i-1, j}+\psi_{i, j+1}+\psi_{i, j-1}\right. \\
& \left.-4 \psi_{i, j}\right)+\frac{\Delta t}{\Delta^{2}}\left(\left[D_{i+1, j}-D_{i, j}\right]\left[\psi_{i+1, j}-\psi_{i, j}\right]\right. \\
& \left.+\left[D_{i, j+1}-D_{i, j}\right]\left[\psi_{i, j+1}-\psi_{i, j}\right]\right)
\end{aligned}
$$

(the time dependence $t$ on the right-hand side of the above has been suppressed in order not to overcrowd the equations), which were then consequently used for all the results shown.
[1] J. Aronowitz and D. Nelson, Phys. Rev. A 29, 2012 (1984).

[2] J. D. Gunton, M. San Miguel, and P. S. Sahni, Phase Trans. 8, 267 (1983).

[3] C. Taylor and J. Cahn, J. Stat. Phys. 77, 183 (1994).

[4] J. Cahn and A. Novickcohen, J. Stat. Phys. 76, 877 (1994).

[5] S. C. Glotzer, D. Stauffer, and N. Jahn, Phys. Rev. Lett. 72, 4109 (1994).

[6] C. C. Ko, T. Kyu, and S. Smith, J. Poly. Sci. B 33, 517 (1995).

[7] A. Vasishta, Chem. Eng. Commun. 129, 29 (1994).

[8] J. M. Liu, Z. G. Liu, and Z. Wu, Chin. Phys. Lett. 11, 634 (1994).

[9] A. M. Lacasta, J. M. Sancho, and F. Sagués, Phys. Rev. Lett. 75, 1791 (1995).
[10] S. Havlin and D. Ben-Avraham, Adv. Phys. 36, 695 (1987).

[11] A. Bunde and S. Havlin, in Fractals and Disordered Systems, edited by S. Havlin and A. Bunde (Springer-Verlag, Berlin, 1991), p. 50.

[12] S. Havlin and A. Bunde, in Fractals and Disordered Systems, (Ref. [11]), p. 96.

[13] M. Saxton, Biophys. J. 66, 394 (1994).

[14] L. O. Landau and E. M. Lifshitz, VIII: Electrodynamics, A Course in Theoretical Physics (Pergamon, Oxford, 1980).

[15] A. Careta, F. Sagués, and J. Sancho, Phys. Fluids 6, 349 (1994). 